\title{
Performance Evaluation of Joint Routing and Scheduling in Opportunistic Sensor Networks
}

\author{
Roohinaz M., PhD \\ Department of Electronics and Communication \\ GCET, Anand, Gujarat, India
}

\author{
Mehul B. Shah \\ Department of Electronics and Communication, \\ GCET, Anand, Gujarat, India
}

\begin{abstract}
In present scenario the innovation of mobile phones that are embedded with different types of onboard sensors, has brought on a new interest of using them as the main part of the sensor network, for a wide area of applications (for e.g. monitoring). In such ad-hoc network end to end connectivity cannot be established and the connection comes out to be mainly opportunistic. The connection in Opportunistic Sensor Network (OSN) is many to one i.e. all the nodes have a common destination. The prominent challenge faced in the opportunistic sensor networks includes the Routing /Scheduling and managing the constrained memory. In this paper scheduling is defined by routing combined with proper buffer management policy and since the scheduling in OSN is node based so selection of proper Buffer management is also significant. This paper considers the monitoring application with the help of human carried mobile sensors and hence a performance evaluation of joint Routing/Scheduling and Buffer management using simulator called ONE (Opportunistic Network Environment) Simulator is done.

Simulation results indicate superiority of Shortest remaining Life Time (SHLI) scheme for node movement as per human mobility characteristics. This is contrary to community mobility model as shown for PROPHET algorithm, in which Most Forwarded First (MOFO) scheme performs better.
\end{abstract}

\section{Keywords}

Opportunistic Sensor Network, Scheduling, Buffer management.

\section{INTRODUCTION}

There is a lot of ambiguity and challenges faced in various places like underwater communication, deep space communication, wildlife monitoring, disaster monitoring and such other places where end to end connectivity can't be easily established. The primitive network solutions cannot be easily applied to such type of networks. It is even seen that the policies pertaining to ad hoc even cannot be applied to them. These network environments are subjected to long delays, persistent disturbances and even limited resources; a category falling under Delay Tolerant Networks (DTN) [1]. One possible way of communication for such type of networks is to go with the approach of store-carry and forward the data and also by exploiting mobility. This situation fall under Opportunistic Sensor Network (OSN) category. The communication in DTN is one to one while in OSN is many to one.

In conventional DTN environment scheduling policies were basically decided based either on network node utility or message utility, that is the decisions were based on which nodes to forward the message or which message to be forwarded. The paradigm shift towards the Opportunistic sensor networks defines scheduling in terms of the routing policy along with proper buffer management schemes [2].

The very recent integration of these sensors with the personal electronic devices like cellular mobile phones has attracted a large number of researchers to consider the appropriate architectures and the applications (i.e., recreational, social) included for large-scale and people-centric sensing systems.

These intermediate mobile nodes implement the store-carryforward message switching mechanism by overlying a new protocol layer, called the bundle layer [3].

The rest of the paper is organized as follows. In Section 2 the system model is presentation. In Section 3, some related work along with the routing protocol PROPHET is described. Section 4 gives a brief description of buffer management and queuing policies which are later on compared in this paper. Section 5 shows the simulation setup while section 6 shows the results that we obtain along with the conclusion.

\section{SYSTEM MODEL}

The work presented in this paper considers the system which draws out inspiration from the OSN environment in which OSN functions are carried out for monitoring purpose.( e.g. Pollution monitoring). In such type of monitoring environment all mobile sensor devices are given the task to sense the pollution data and upload them over a common Access Point or Pollution board data base [5].

For the above mentioned scenario the proposed sensors are human carried sensors. In such case human mobility is what we are concerned about for an OSN environment. By exploiting this mobility issue, a new communication opportunity can be created among otherwise network elements that are isolated. So the work proposed here highlights such type of applications like Pollution Monitoring in which exchange of data can only take place in delay fashion, while the nodes are in range. The concerns here include, lack of available resources, which including storage space, execution and processing of memory, and transmission power.

Thus taking into consideration one of the above problem i.e. limited storage space compels to find a solution for managing the buffer space available with each of the mobile nodes. This concept lead to jointly use the routing and buffer management for opportunistic sensor network environment [2]. In this paper evaluation of the performance of a set of queuing policies is combined with probabilistic routing, for human mobility. Comparison of the performance of set of these 
different strategies for two mobility models named Levy Walk and Self similar Least Action Walk (SLAW) is done.

\section{RELATED WORK}

The authors in [8] have discussed the evaluation of various queuing policies along with different forwarding strategies for intermittently connected network falling under DTN. They have compared two routing protocols and then showed the corresponding results. The authors have carried out the proposed work using a community based mobility model.

In [11] the authors have proposed the human mobility model called truncated levy walk [12]. The different traces have been generated by changing the value of parameters called the levy exponent $\alpha$ and $\beta$ so that proposed model fit to the actual movement patterns of human beings. In [13] the authors have proposed another mobility model called SLAW. Here they have generated the traces from the human walking patterns at common gathering places which people visit the most daily. They have generated so using the fractal points and heavytailed flights waypoints.

\section{PROBABILISTIC ROUTING PROTOCOL}

In [10] a routing protocol which exploits mobility patterns of users has been proposed. In real time users are not likely to move around in a random fashion, but rather they move around in a predictable fashion which is based on repeating several behavioral patterns in such a way that if a node has visited a particular location several times before, it is possible that it will visit that particular location again. Based on this fashion of repetition probability factor is calculated in [10] and decision is made on to which node the packet should be forwarded.

The predictable delivery also follows a property of transitivity, which is likely based on the observation that if there is node $X$ that very often encounters node $Y$, and there is node $\mathrm{Y}$ that often encounters node $\mathrm{Z}$, then node $\mathrm{Z}$ is probably a valid node in order to forward messages which are destined for node $\mathrm{X}$.

The calculation parameters of the delivery predictions have following three parts. The foremost thing that has to be done is to update the calculated metric whenever a node is encountered, such that the hosts that are likely to be encountered most probably have very high delivery predictability. The calculation that is shown in Eq. 1, where Pinit $\in[0,1]$ shows the value of initialization constant.

$$
\mathrm{P}_{(\mathrm{a}, \mathrm{b})}=\mathrm{P}_{(\mathrm{a}, \mathrm{b}) \text { old }}+\left(1-\mathrm{P}_{(\mathrm{a}, \mathrm{b}) \text { old }}\right) \times \mathrm{P}_{\text {init }}
$$

If the shown pair of nodes will not meet each other in a defined time, they are not to be called the ones who can forward all of the messages to each other, and thus the delivery predictability vector values should age, which are being reduced in the process. This aging equation is shown in Eq. 2, where $\gamma \in[0,1)$ is called the aging constant, and where $\mathrm{k}$ shows the number of time units that might have elapsed since the last time the metric was aged.

$$
P_{(a, b)}=P_{(a, b) o l d} \times \gamma^{k}
$$

The predictable delivery also follows a property of transitivity. Eq. 3 shows that how this transitivity affects the delivery predictability, here $\beta \in[0,1]$ is called scaling constant which decides that how large impact the transitivity property should have in order to rely on the delivery predictability vector.

$$
\mathrm{P}_{(\mathrm{a}, \mathrm{c})}=\mathrm{P}_{(\mathrm{a}, \mathrm{c}) \text { old }}+\left(1-\mathrm{P}_{(\mathrm{a}, \mathrm{c}) \text { old }}\right) \times \mathrm{P}_{(\mathrm{a}, \mathrm{b})} \times \mathrm{P}_{(\mathrm{b}, \mathrm{c})} \times \beta
$$

\section{BUFFER MANAGEMENT AND QUEUING POLICIES}

When the node's buffer is full, then often in order to accommodate a new message, node has to drop an important message. If an efficient policy of dropping is implemented that can help prioritize the message drop sequence. This will give a huge impact on the delivery ratio present in the network. It's not just dropping policies that define buffer management in, but also the scheduling policies matter. In OSN proper scheduling is defined by routing combined with proper buffer management policy. Moreover routing decisions are node based, so selection of proper Buffer management is also significant. Nodes have to buffer messages for a long time and in case of network congestion they have to decide which messages to drop from its queue. In this section we describe the different queuing policies [18] used in this paper for the performance evaluation in Section 5 .

FIFO: First in First Out.

The node is strategized in the order of first in first out. So the message which is queued first is dropped first.

LIFO: Last in First Out

The node in this policy is strategized in the order of last in first out. So the message which is queued last is the first one to be dropped.

MOFO: Evict Most Forwarded first

The message that is forwarded most number of times is dropped first in order to the give the less forwarded messages the chance to be forwarded. The node has to keep a track on number of times the message will be forwarded.

SHLI: Evict Shortest lifetime first

Every message existing in the network has a time out value. After the time has exceeded the message is no longer useful and should be discarded. Thus here the message with the shortest lifetime is dropped first.

\section{SIMULATION SETUP}

Java based simulator called ONE (Opportunistic Network Environment Simulator) [19] is done. The simulation is done for evaluating performance of joint Routing/Scheduling and four different Buffer management schemes FIFO, LIFO, SHLI, and MOFO.

All the results here are evaluated under human mobility model as this is the realistic mobility for OSN. In some of the previous works the authors have used mobility models like random way point or mobility data gathered from real life measurements. Some have even used community model so that they could calculate results for scene which is relatively close to the characteristics of human mobility. The simulation area here is $500 \times 500$ meters. The simulation time set up is 28800 seconds. The total number of nodes taken into consideration here is 15 mobile nodes destined to send messages to a single sink. Message generation takes place after every second. In this work evaluation of the performance is done by generating total 1000 messages all of different sizes varying from $500 \mathrm{~Kb}$ to $999 \mathrm{~Kb}$. This range is chosen because in our set up we have considered the buffer size to be 
1M. The message size range can be changed along with appropriate buffer size. In the first environment the results are plotted by varying the transmission range. During the first 10 meter there is hardly any change seen for the different queuing strategies. But after 20 meter, changes are more prominent. The transmission range is varied to $\{20 \mathrm{~m}, 30 \mathrm{~m}$, and $40 \mathrm{~m}\}$; the message TTL for this case is kept to be infinite. The buffer size is kept $1 \mathrm{M}$. For the second scenario the transmission range is fixed to 30 meter and ttl to 300 minutes and varied the buffer size to $1 \mathrm{M}, 2 \mathrm{M}$ and $3 \mathrm{M}$. We have considered this value of range and ttl because we get more distinguished results by keeping this value. In our third setup we have varied the message ttl to 400 minutes, 500 minutes and 600 minutes and observed the changes by fixing the buffer size to $1 \mathrm{M}$ and transmission range to 30 meter. The performance metrics for which the results are shown below.

\subsection{Performance Metrics}

\section{Delivery Probability}

The delivery probability metric shows the probable number of messages that have been transmitted from the total number of messages available.

\section{$>\quad$ Average Latency}

The average latency is the average time delay during when the period when the message was delivered and received.

\subsection{Simulation Results}

First a total of 10 mobility traces have been generated using synthetic mobility models called TLW and SLAW and imported in the simulator. The values of performance metrics is averaged over these 10 traces.

\begin{tabular}{|c|c|}
\hline $\begin{array}{l}\text { RESULTS-1 } \\
\text { SIMULATION } \\
\text { PARAMETERS }\end{array}$ & VALUES \\
\hline Simulation time & $28800 \mathrm{~s}$ \\
\hline No of nodes & 16 \\
\hline Routing Protocol & Prophet \\
\hline Mobility Model & TLW, SLAW \\
\hline Transmission Range & $20 \mathrm{~m}, 30 \mathrm{~m}, 40$ \\
\hline Buffer size & $1 \mathrm{M}$ \\
\hline TTL & Infinite \\
\hline
\end{tabular}

Table 1. Simulation parameters-1

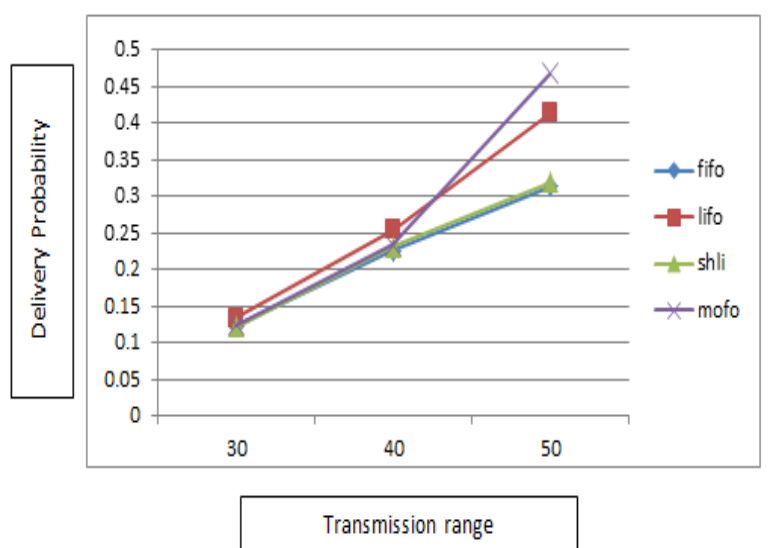

Fig (a) Delivery Probability vs Trans. range for Levy Walk

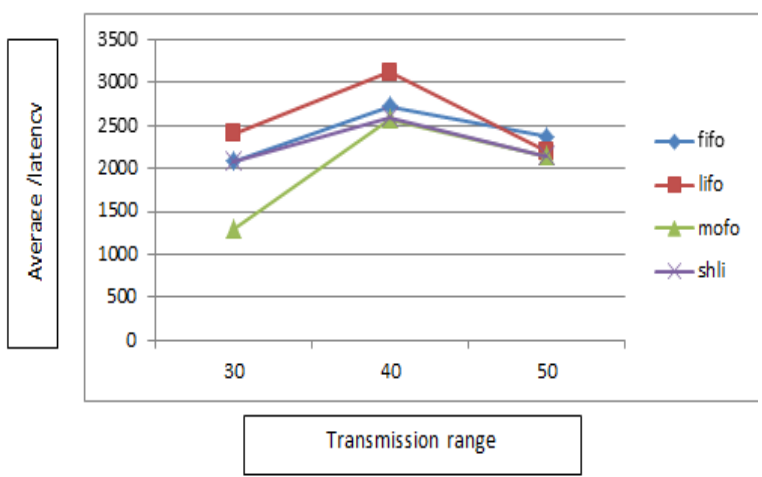

Fig (b) Average Latency vs Transmission range for Levy Walk

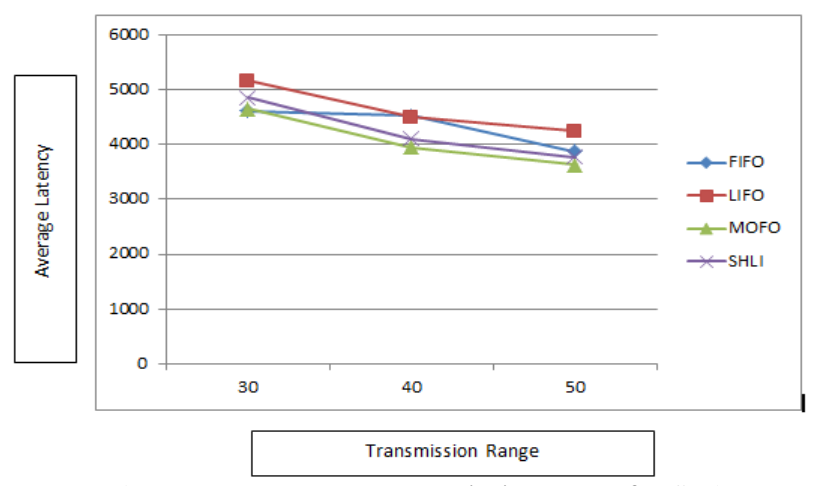

Fig (c) Average Latency vs Transmission range for SLAW

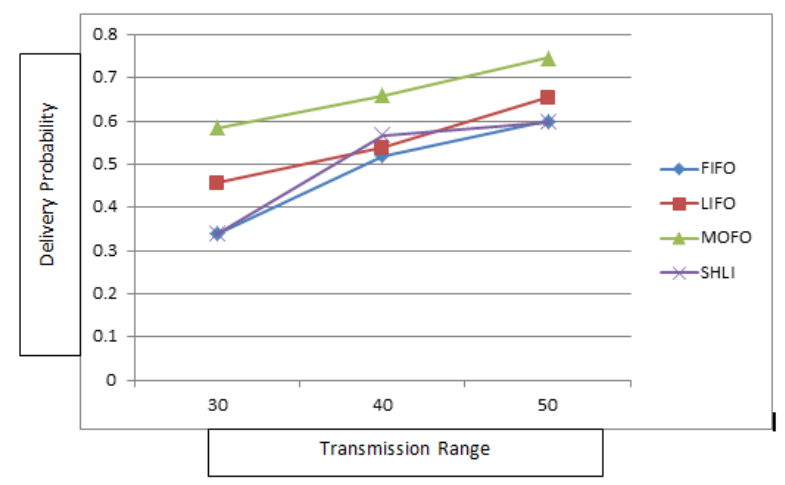

Fig (d) Delivery Probability vs Transmission range for SLAW

- In the first simulation setup the performance evaluation of four buffer management policies namely FIFO (First in First Out), LIFO (Last in First Out), MOFO (Most Forwarded First) and SHLI (Shortest life first) is done by varying three different transmission ranges. From the simulation results it could be seen that the buffer management policy of MOFO shows better results in comparison to the other policies for both the performance metrics of delivery probability and Average Latency. Since MOFO drops the most number of times forwarded messages in the network, it takes care that the message that are spread most in the network are dropped. Due to this as the distance increases it is made sure that the messages are forwarded at least once to retain their availability in the network. Hence the delivery probability increases with increase in the distance. 
- RESULTS-2

\begin{tabular}{|c|c|}
\hline $\begin{array}{c}\text { SIMULATION } \\
\text { PARAMETERS }\end{array}$ & VALUES \\
\hline Simulation time & $28800 \mathrm{~s}$ \\
\hline No of nodes & 16 \\
\hline Routing Protocol & Prophet \\
\hline Mobility Model & TLW, SLAW \\
\hline Transmission Range & $30 \mathrm{~m}$ \\
\hline Buffer size & $1 \mathrm{M}, 2 \mathrm{M}, 3 \mathrm{M}$ \\
\hline TTL & $300 \mathrm{mins}$ \\
\hline
\end{tabular}

Table 2. Simulation parameters-2

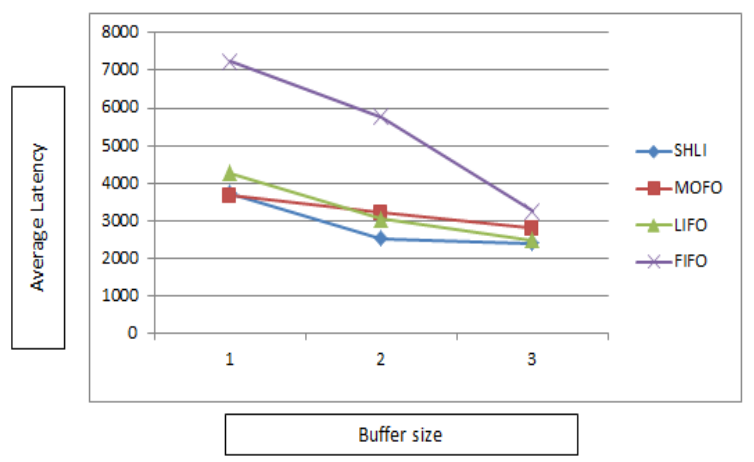

Fig(e) Average latency vs Buffer Size for Levy Walk

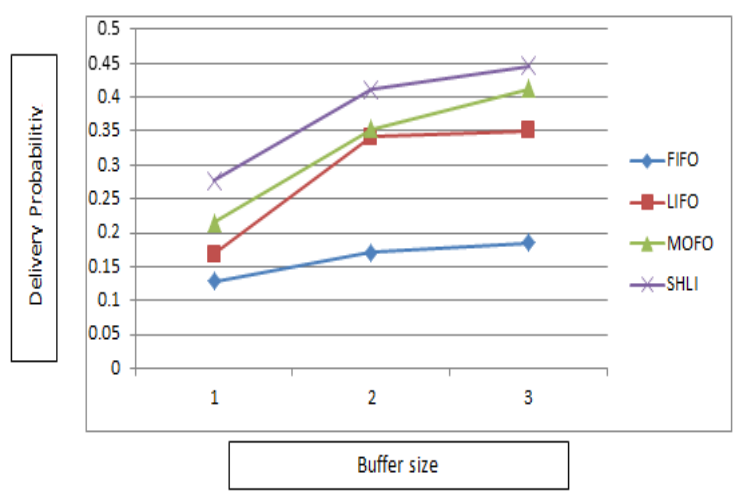

Fig(f) Delivery Probability vs Buffer Size for Levy Walk

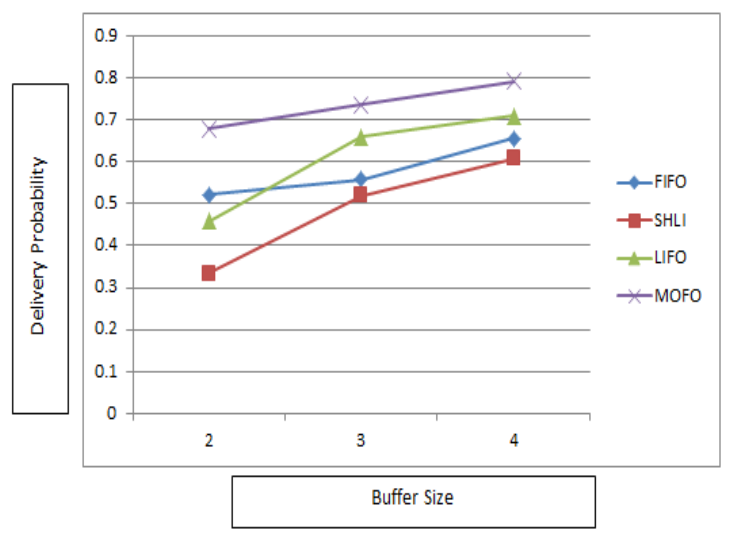

Fig(g) Delivery Probability vs Buffer Size for SLAW

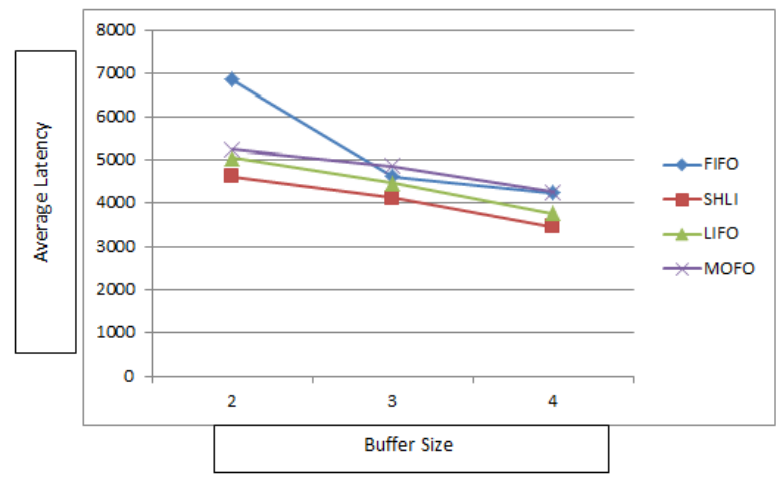

Fig(h) Average latency vs Buffer Size for SLAW

- In the second simulation setup performance evaluation of four buffer management policies namely FIFO (First in First Out), LIFO (Last in First Out), MOFO (Most Forwarded First) and SHLI (Shortest life first) by varying three different buffer values. From the graph obtained by varying the average latency vs. Buffer size, SHLI shows better performance. In the second graph of delivery probability vs. varying buffer size again SHLI exhibits better performance. SHLI drops the message that has the lowest ttl value so for corresponding buffer sizes it will quickly deliver the message before its ttl expires and probability of delivery increases as shown in following figures. While MOFO seems to be giving better performance in case of Delivery probability and SHLI for Average Latency in case of SLAW.

\section{- RESULTS-3}

\begin{tabular}{|c|c|}
\hline $\begin{array}{c}\text { SIMULATION } \\
\text { PARAMETERS }\end{array}$ & VALUES \\
\hline Simulation time & $28800 \mathrm{~s}$ \\
\hline No of nodes & 16 \\
\hline Routing Protocol & Prophet \\
\hline Mobility Model & TLW, SLAW \\
\hline Transmission Range & $20 \mathrm{~m}, 30 \mathrm{~m}, 40$, \\
\hline Buffer size & $1 \mathrm{M}$ \\
\hline TTL & $400,500,600$ (mins) \\
\hline
\end{tabular}

Table 3. Simulation parameters-3

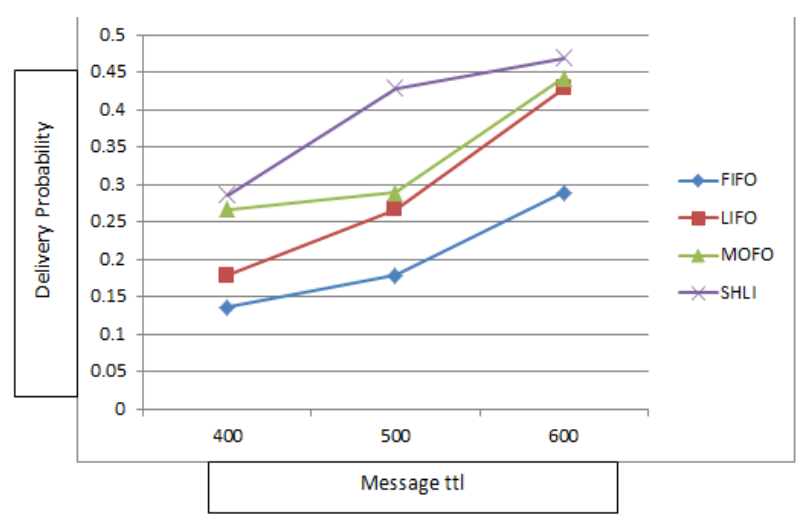

Fig(i) Delivery Probability vs Message TTL for Levy Walk 


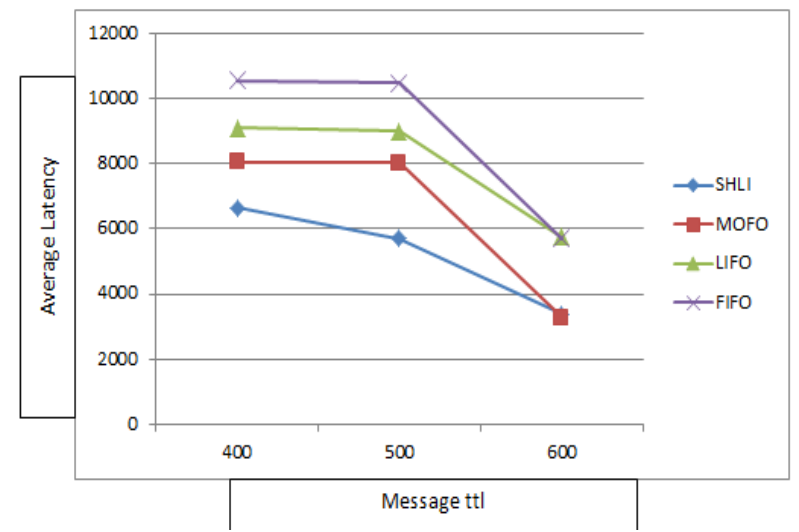

Fig(j) Average latency vs Message TTL for Levy Walk

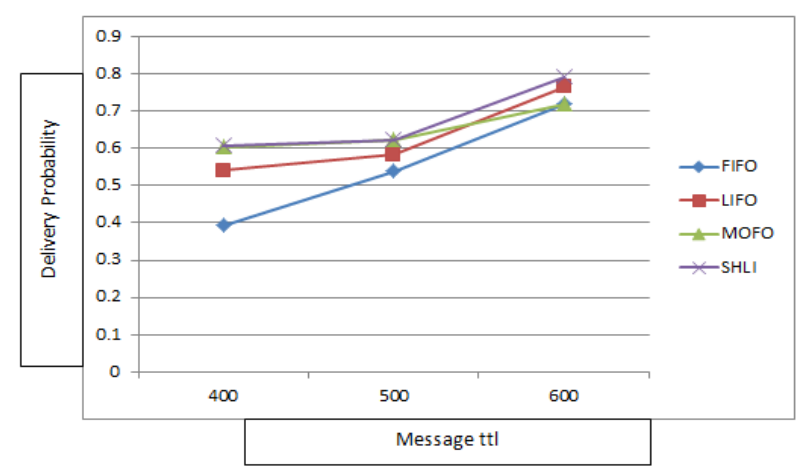

Fig(k) Delivery Probability vs Message TTL for SLAW

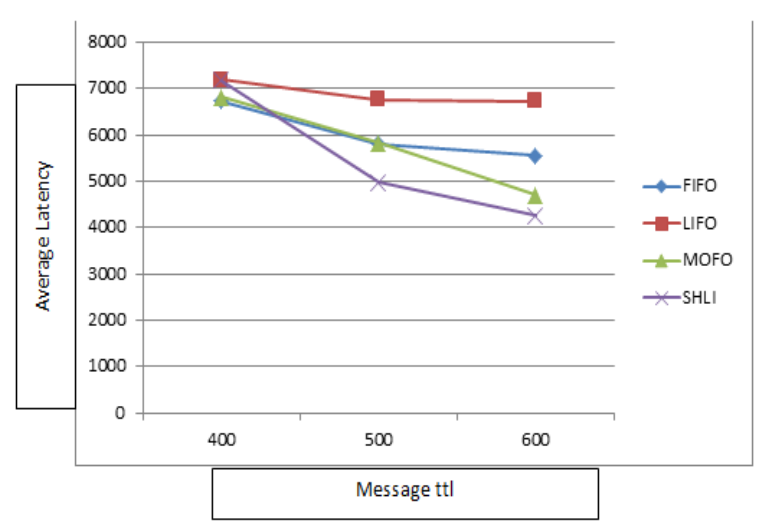

Fig(1) Average latency vs Message TTL for SLAW

- In the third simulation setup the performance evaluation of four buffer management policies namely FIFO (First in First Out), LIFO (Last in First Out), MOFO (Most Forwarded First) and SHLI (Shortest life first) by varying three different $\mathrm{ttl}$ (message time to live) values. The graph of delivery probability here shows that the buffer management policy of SHLI shows better performance results.

The graph obtained for average latency vs. TTL value shows that the buffer management policy of SHLI shows comparatively better performance for both the mobility models. Here probably before the message expires it is quickly delivered, hence increasing the delivery probability.

\section{CONCLUSION}

In this paper evaluation of the performance of various buffer management policies under human mobility is done. Using it along with probabilistic routing shows better results than other flooding approach in routing. Resources like buffer space needs to be managed properly because its inclusion is inevitable in all sensing devices. Human carried devices such as cell phone have limited buffer size as it is used for multiple applications.

The result shows superiority of SHLI scheme for node movement as per Human mobility along with PROPHET. MOFO scheme was observed to perform better along with PROPHET for random node movements. This observation is contrary to the results obtained with random node movements along with PROPHET protocol [8].

\section{REFERENCES}

[1] Yick, Jennifer, Biswanath Mukherjee, and Dipak Ghosal. "Wireless sensor network survey." Computer networks 52.12 (2008): 2292-2330.

[2] Krifa, Amir, Chadi Barakat, and Thrasyvoulos Spyropoulos. "Message drop and scheduling in DTNs: Theory and practice." Mobile Computing, IEEE Transactions on 11.9 (2012): 1470-1483.

[3] Chung-Ming Huang, Kun-chan Lan and Chang-Zhou Tsai "A survey on Opportunistic Sensor Network" International conference on advanced Information Networking and Applications IEEE, 2008.292

[4] Le, Viet-Duc, J. Scholten, and P. J. M. Havinga. "Evaluation of opportunistic routing algorithms on opportunistic mobile sensor networks with infrastructure assistance." International Journal On Advances in Networks and Services 5.3-4 (2012): 279-290.

[5] Eisenman, Shane B., Hong Lu, and Andrew T. Campbell. "Halo: Managing node rendezvous in opportunistic sensor networks." Distributed Computing in Sensor Systems. Springer Berlin Heidelberg, 2010. 273-287.

[6] Shah, Mehul B., Shabbir N. Merchant, and Uday B. Desai. "Human-Mobility-Based Sensor Context-Aware Routing Protocol for Delay-Tolerant Data Gathering in Multi-Sink Cell-Phone-Based Sensor Networks." International Journal of Distributed Sensor Networks 2012 (2012)

[7] Soares, Jorge M., et al. "Opportunistic data collection in sparse wireless sensor networks." Eurasip Journal on Wireless Communications and Networking 2011 (2011): 6.

[8] Lindgren, Anders, and Kaustubh S. Phanse. "Evaluation of queueing policies and forwarding nication System Software and Middleware, 2006. Comswastrategies for routing in intermittently connected networks."Commure 2006. First International Conference on. IEEE, 2006.

[9] Huang, Chung-Ming, Kun-chan Lan, and Chang-Zhou Tsai. "A survey of opportunistic networks." Advanced Information Networking and Applications-Workshops, 2008. AINAW 2008. 22nd International Conference on. IEEE, 2008.

[10] Lindgren, Anders, Avri Doria, and Olov Schelén. "Probabilistic routing in intermittently connected networks." ACM SIGMOBILE mobile computing and communications review 7.3 (2003): 19-20. 
[11] M Shahzamal, M F Pervez, M A U Zaman and M D Hossain Mobility Models For Delay Tolerant Network: A Survey, International Journal of Wireless \& Mobile Networks (IJWMN) Vol. 6, No. 4, August 2014

[12] Rhee, I., Shin, M., Hong, S., Lee, K., Kim, S. J., \& Chong, S. (2011). On the levy-walk nature of human mobility. IEEE/ACM transactions on networking (TON), 19(3), 630-643.

[13] Lee, Kyunghan, et al. "Slaw: A new mobility model for human walks."INFOCOM 2009, IEEE. IEEE, 2009.

[14] Khabbaz, Maurice J., Chadi M. Assi, and Wissam F. Fawaz. "Disruption-tolerant networking: A comprehensive survey on recent developments and persisting challenges." Communications Surveys \& Tutorials, IEEE 14.2 (2012): 607-640.

[15] Ari Ker"anen, Teemu K"arkk"ainen, and Jorg Ott, Simulating Mobility and DTNs with the ONE, Journal of communication, 2010
[16] Mayer, Christoph P., and Oliver P. Waldhorst. "Routing in hybrid delay tolerant networks." Computer Communications 48 (2014): 44-55.

[17] Jain, Sweta, and Meenu Chawla. "Survey of buffer management policies for delay tolerant networks." The Journal of Engineering 1.1 (2014).

[18] Keränen, Ari, Jörg Ott, and Teemu Kärkkäinen. "The ONE simulator for DTN protocol evaluation." Proceedings of the 2nd international conference on simulation tools and techniques. ICST (Institute for Computer Sciences, Social-Informatics and Telecommunications Engineering), 2009.

[19] Eisenman, Shane B., Nicholas D. Lane, and Andrew T. Campbell. "Techniques for improving opportunistic sensor networking performance." Distributed Computing in Sensor Systems. Springer Berlin Heidelberg, 2008. 157-175. 